\title{
Geodesic balls in a fuzzy set and fuzzy geodesic mathematical morphology
}

\author{
Isabelle Bloch* \\ Ecole Nationale Supérieure des Télécommunications, Département TSI - CNRS URA 820, 46 rue Barrault, 75013 Paris, France
}

Received 23 July 1998; received in revised form 21 December 1998; accepted 2 May 1999

\begin{abstract}
Although fuzzy operators have deserved a large attention in the Euclidean case, almost nothing exists concerning the geodesic case. In this paper, we address this question, by defining fuzzy geodesic distances between points in a fuzzy set, and geodesic balls in a fuzzy set (based on the comparison of fuzzy numbers), from which we derive fuzzy geodesic mathematical morphology operators. The proposed definitions are valid in any dimension. The main properties of the basic operators are demonstrated. These new operations enhance the set of fuzzy morphological operators, leading to transformations of a fuzzy set conditionally to another fuzzy set. (C) 2000 Pattern Recognition Society. Published by Elsevier Science Ltd. All rights reserved.
\end{abstract}

Keywords: Fuzzy sets; Fuzzy geodesic distance; Fuzzy geodesic balls; Fuzzy mathematical morphology; Fuzzy geodesic dilation and erosion

\section{Introduction}

The extension of mathematical morphology to fuzzy sets has become a focus of interest in several research teams since a few years, e.g. Refs. [1-7] and several others since these original works. One interesting point of view of these extensions relies in the links existing between fuzzy morphological operators (in particular distances) and fuzzy distances. For instance, in previous works [8-10], we have shown how fuzzy morphological operators can be derived from fuzzy distances, and conversely how fuzzy dilation can be the basis for powerful fuzzy distances between a point and a fuzzy set and between two fuzzy sets.

Such links are widely studied in classical morphology, in the Euclidean case, but also in the geodesic case as well. Indeed, in mathematical morphology, an important set of operations is constituted by geodesic transformations [11-14]. They are most useful in image processing

\footnotetext{
* Tel.: + 33(1)-45-81-75-85; fax: + 33(1)-45-81-37-94

E-mail address: isabelle.bloch@enst.fr (I. Bloch)
}

and pattern recognition, where transformations may have to be performed conditionally to a restriction of the spatial domain. Applications can be found for defining operators under reconstruction (e.g. filtering operators), in image segmentation, and in pattern recognition, where operations have to be constrained by results of some other transformations.

In this paper, we propose to define geodesic transformations on fuzzy sets, that extend our preliminary work in Ref. [15]. To our knowledge, this is the first attempt towards extending geodesic morphology to fuzzy sets, in contrary to Euclidean morphology, that has already motivated several works [1-7]. The aim of this extension is to provide geodesic operators for image processing under imprecision, where image objects are represented as spatial fuzzy sets. An object in the image is represented as a fuzzy set through a membership function assigning to each point of the image a value in $[0,1]$ which represents its membership degree to the object. ${ }^{1}$

\footnotetext{
${ }^{1}$ What is called object depends on the application. It may be for instance a region in the image to which we can assign a label or a semantics.
} 
With such a representation, spatial imprecision, for instance on the limits of the objects, is directly taken into account. We will consider mainly dilation and erosion, which are the two main morphological operators, from which a large set of operators can be built, by iterating and combining these two basic ones.

Let us first introduce some notations and recall some definitions of geodesic morphology on binary sets. In the Euclidean case, the considered space $\mathscr{S}$ is equipped with the Euclidean distance $d_{E}$, and we denote by $D^{r}(Y)$ and $E^{r}(Y)$ the dilation and erosion of a set $Y$ by a ball $B_{r}$ of size $r$. In the geodesic case, transformations are defined conditionally to a reference set $X$. The considered distance is then the geodesic distance in $X$ (i.e. the distance $d_{X}(x, y)$ from $x$ to $y$ is the length of the shortest path from $x$ to $y$ completely included in $X$ ). A geodesic ball of size $r$ and center $x$ is defined as

$B_{X}(x, r)=\left\{y \in X, d_{X}(x, y) \leqslant r\right\}$.

Geodesic dilation and erosion of $Y$ conditionally to $X$ of size $r$ are then defined as

$$
\begin{aligned}
D_{X}^{r}(Y) & =\left\{x \in \mathscr{S}, B_{X}(x, r) \cap Y \neq \emptyset\right\} \\
& =\left\{x \in \mathscr{S}, d_{X}(x, Y) \leqslant r\right\}, \\
E_{X}^{r}(Y) & =\left\{x \in \mathscr{S}, B_{X}(x, r) \subset Y\right\}=X-D_{X}^{r}(X-Y) .
\end{aligned}
$$

We propose to generalize Eqs. (1)-(3) to fuzzy sets. We first define the type of fuzzy sets we use here in Section 2, and present a general principle for extending operations. The generalization of Eqs. (1)-(3) to fuzzy sets calls for extensions of geodesic distance and of geodesic balls to fuzzy sets. We have already proposed several definitions for fuzzy geodesic distances in Ref. [16]. We recall the definition having the best properties in Section 3, and propose another definition where the distance is considered as a fuzzy number. We propose in Section 4 a definition of fuzzy geodesic balls and we give its main properties. In Section 5 we derive definitions of fuzzy geodesic dilation and erosion, and present their algebraic properties.

\section{Spatial fuzzy sets and extension of operations}

A useful representation of objects in images under imprecision can be found in the framework of fuzzy sets $[17,18]$. The space $\mathscr{S}$ is the image space, typically $\mathbb{Z}^{2}$ or $\mathbb{Z}^{3}$ for digital $2 \mathrm{D}$ or $3 \mathrm{D}$ images, or, in the continuous case, $\mathbb{R}^{2}$ or $\mathbb{R}^{3}$. We are interested in the objects of the image that we may describe as fuzzy sets. Thus we often call them fuzzy image objects. A fuzzy image object is a fuzzy set defined on $\mathscr{S}$, i.e. a spatial fuzzy set. Its membership function $\mu$ is a function from $\mathscr{S}$ into $[0,1]$ and represents the imprecision in the spatial extent of the object. For any point $x$ of $\mathscr{S}$ (pixel or voxel), $\mu(x)$ is the

degree to which $x$ belongs to the fuzzy object. Since it is equivalent to speak about a fuzzy set or its membership function, we will use in the following either of both terms, and denote both by $\mu$.

The advantage of this representation is to account for spatial imprecision that is inherent to images in several domains. This imprecision may originate from the observed phenomenon itself, from the limited resolution, from the reconstruction algorithms, etc. [18]. Spatial fuzzy sets therefore represent both the spatial information and the imprecision attached to it.

When dealing with fuzzy objects, operations usually defined on crisp (or classical or binary) sets have to be extended to fuzzy objects. Several different methods have been proposed in the literature to this aim [2,19-21]. The method we use here consists in translating binary expressions into fuzzy ones. This method is particularly powerful if the operations can be expressed in set theoretical or logical terms.

The idea is to replace formally every binary (or crisp) concept by its fuzzy equivalent. Table 1 summarizes the main definitions of fuzzy equivalents (the reader may find more details about definitions and properties of t-norms, t-conorms and complementations in Refs. [22-24]).

From these equivalences, more complex relationships can be translated. For instance, the expression $A \subset B$, which is equivalent to $A^{C} \cup B=\mathscr{S}$, is translated as

$\inf _{x \in \mathscr{S}} T\left[c\left(\mu_{A}\right)(x), \mu_{B}(x)\right]$

which is a number in $[0,1]$ representing the degree to which the fuzzy set $\mu_{A}$ is included in the fuzzy set $\mu_{B}$. The functions $\mu_{A}$ and $\mu_{B}$ represent the two concerned fuzzy sets, or equivalently their membership functions.

Such translations have already been used for defining Euclidean morphological operators [2], leading to the following generic expressions for the dilation and erosion of a fuzzy set $\mu$ by a fuzzy structuring element $v$ :

$$
\begin{aligned}
& \forall x \in \mathscr{S}, D(\mu, v)(x)=\sup _{y \in \mathscr{S}} t[\mu(y), v(y-x)], \\
& \forall x \in \mathscr{S}, E(\mu, v)(x)=\inf _{y \in \mathscr{S}} T[\mu(y), c(v(y-x))] .
\end{aligned}
$$

Table 1

Crisp concept

Set X

Characteristic function

$\mu, \mu(x) \in\{0,1\}$

Complement of a set

Intersection $\cap$

Union $\cup$

Existence $\exists$

Universal symbol $\forall$
Equivalent fuzzy concept

Fuzzy set

Membership function

$\mu, \mu(x) \in[0,1]$

Fuzzy complementation $c$

t-norm $t$

t-conorm $T$

Supremum

Infimum 
These definitions have good properties in terms of both mathematical morphology and fuzzy sets, as shown in Ref. [2]. Therefore, we based our work on these definitions. The proposed construction of geodesic operators will follow the same principle (Section 5).

One of the main advantages of this construction principle is that it leads to a nice axiomatization of the resulting operations. Indeed, since the fuzzy equivalent concepts of the basic set and logical operations share most of the properties of these crisp operations, the derived complex operations also satisfy a set of axioms. This set is precisely the one that has to be satisfied in order to share similar properties in the fuzzy case and in the crisp case. However, as can be expected from any extension, some properties may be lost. The amount of loss depends on the choice of the t-norms and t-conorms. For instance, for Euclidean fuzzy morphology defined as in Eqs. (4) and (5), most properties of the operations are satisfied whatever the choice of the t-norms and tconorms. A few properties are satisfied only for specific choices of these connectives. This is the case for instance for the idempotence of opening and closing, that is satisfied only for the Lukasiewicz t-norm and t-conorm (i.e. $t(a, b)=\max (0, a+b-1)$ and $T(a, b)=\min (1, a+b))$ [2].

\section{Fuzzy geodesic distance between two points in a fuzzy set}

\subsection{Fuzzy geodesic distance defined as a number}

We proposed in Ref. [16] an original definition for the distance between two points in a fuzzy set, extending the notion of geodesic distance. We recall here this definition and the main results we obtained.

The geodesic distance between two points $x$ and $y$ represents the length of the shortest path between $x$ and $y$ that "goes out of $\mu$ as least as possible". We have proposed several formalisms for this notion. Here we recall only the one having the best properties. This definition relies on the degree of connectivity, as defined by Rosenfeld [25]. In the case where $\mathscr{S}$ is a discrete bounded space (as is usually the case in image processing), the degree of connectivity in $\mu$ between any two points $x$ and $y$ of $\mathscr{S}$ is defined as

$$
c_{\mu}(x, y)=\max _{L_{i} \in L}\left[\min _{t \in L_{i}} \mu(t)\right],
$$

where $L$ denotes the set of all paths from $x$ to $y$. Each possible path $L_{i}$ from $x$ to $y$ is constituted by a sequence of points of $\mathscr{S}$ according to the discrete connectivity defined on $\mathscr{S}$.

We denote by $L^{*}(x, y)$ a shortest path between $x$ and $y$ on which $c_{\mu}$ is reached (this path, not necessarily unique, can be interpreted as a geodesic path descending as least as possible in the membership degrees), and we denote by $l\left(L^{*}(x, y)\right)$ its length (computed in the discrete case from the number of points belonging to the path). Then we define the geodesic distance in $\mu$ between $x$ and $y$ as

$d_{\mu}(x, y)=\frac{l\left(L^{*}(x, y)\right)}{c_{\mu}(x, y)}$.

If $c_{\mu}(x, y)=0$, we have $d_{\mu}(x, y)=+\infty$, which corresponds to the result obtained with the classical geodesic distance in the case where $x$ and $y$ belong to different connected components (actually it corresponds to generalized geodesic distance, where infinite values are allowed).

This definition corresponds to the weighted geodesic distance (in the classical sense) computed in the $\alpha$-cut of $\mu$ at level $\alpha=c_{\mu}(x, y)$. In this $\alpha$-cut, $x$ and $y$ belong to the same connected component (for the considered discrete crisp connectivity). This definition is illustrated in Fig. 1.

This definition satisfies the following set of properties (see Ref. [16] for the proof):

(1) positivity: $\forall(x, y) \in \mathscr{S}^{2}, d_{\mu}(x, y) \geqslant 0$;

(2) symmetry: $\forall(x, y) \in \mathscr{S}^{2}, d_{\mu}(x, y)=d_{\mu}(y, x)$;

(3) separability: $\forall(x, y) \in \mathscr{S}^{2}, d_{\mu}(x, y)=0 \Leftrightarrow x=y$;

(4) $d_{\mu}$ depends on the shortest path between $x$ and $y$ that "goes out" of $\mu$ "as least as possible", and $d_{\mu}$ tends towards infinity if it is not possible to find a path between $x$ and $y$ without going through a point $t$ such that $\mu(t)=0$

(5) $d_{\mu}$ is decreasing with respect to $\mu(x)$ and $\mu(y)$;

(6) $d_{\mu}$ is decreasing with respect to $c_{\mu}(x, y)$;

(7) $d_{\mu}$ is equal to the classical geodesic distance if $\mu$ is crisp.

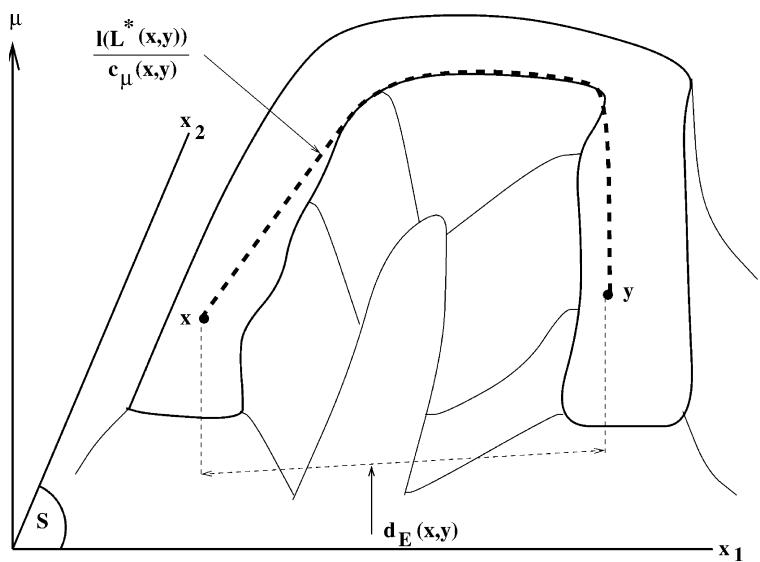

Fig. 1. Illustration of the geodesic distance in a fuzzy set $\mu$ between two points $x$ and $y$ in a 2D space. 
The triangular inequality is not satisfied, but from this definition, it is possible to build a true distance, satisfying triangular inequality, while keeping all other properties. This can be achieved in the following way (see Ref. [16] for proof and details):

$d_{\mu}^{\prime}(x, y)=\min _{t \in \mathscr{S}}\left[\frac{l\left(L^{*}(x, t)\right)}{c_{\mu}(x, t)}+\frac{l\left(L^{*}(t, y)\right)}{c_{\mu}(t, y)}\right]$

These properties are in agreement with what can be required from a fuzzy geodesic distance, both mathematically and intuitively.

\subsection{Fuzzy geodesic distance defined as a fuzzy number}

In the previous approach, the geodesic distance between two points is defined as a crisp number (i.e. a standard number). It could be also defined as a fuzzy number, taking into account the fact that, if the set is imprecisely defined, geodesic distances in this set can be imprecise too. This is the scope of this section.

One solution to achieve this aim is to use the extension principle, based on a combination of the geodesic distances computed on each $\alpha$-cut of $\mu$. Let us denote by $d_{\mu_{x}}(x, y)$ the geodesic distance between $x$ and $y$ in the crisp set $\mu_{\alpha}$. Using the extension principle, we define the degree to which the geodesic distance between $x$ and $y$ in $\mu$ is equal to $d$ as

$\forall d \in \mathbb{R}^{+}, d_{\mu}(x, y)(d)=\sup \left\{\alpha \in[0,1], d_{\mu_{\alpha}}(x, y)=d\right\}$.

This definition satisfies the following properties:

(1) If $\alpha>c_{\mu}(x, y)$, then $x$ and $y$ belong to two distinct connected components of $\mu_{\alpha} \cdot{ }^{2}$ In this case, the (generalized) geodesic distance is infinite. If we restrict the evaluation of $d_{\mu}(x, y)(d)$ to finite distances $d$, then $d_{\mu}(x, y)(d)=0$ for $d>d_{\mu_{c u(x, y)}}$.

(2) Let $d_{E}(x, y)$ denote the Euclidean distance between $x$ and $y$. It is the shortest of the geodesic distances that can be obtained in any crisp set that contains $x$ and $y$. This set can be for instance the whole space $\mathscr{S}$, which can be assimilated to the $\alpha$-cut of level $0\left(\mu_{0}\right)$. Therefore, for $d<d_{E}(x, y)$, we have $d_{\mu}(x, y)(d)=0$.

(3) Since the $\alpha$-cuts are nested $\left(\mu_{\alpha} \subset \mu_{\alpha^{\prime}}\right.$ for $\left.\alpha>\alpha^{\prime}\right)$, it follows that $d_{\mu_{x}}(x, y)$ is increasing in $\alpha$, for $\alpha \leqslant c_{\mu}(x, y)$. Therefore, $d_{\mu}(x, y)$ is a fuzzy number, with a maximum value for $d_{\mu_{c u(x, y)}}$, and with a discontinuity at this point. Its shape looks as shown in Fig. 2.

\footnotetext{
${ }^{2}$ Since $c_{\mu}(x, y)$ corresponds to "height" (in terms of membership values) of the point along the path that connects $x$ and $y$, i.e. the maximum of the minimal height along paths from $x$ to $y$.
}

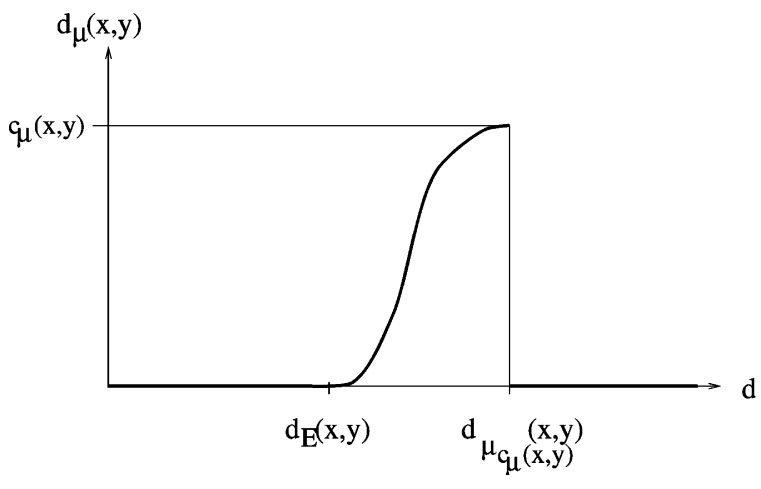

Fig. 2. Typical shape of the fuzzy geodesic distance between two points in a fuzzy set, defined as a fuzzy number.

This definition can be normalized by dividing all values by $c_{\mu}(x, y)$, in order to get a maximum membership value equal to 1 .

One drawback of this definition is the discontinuity at $d_{\mu_{c(x, y, y)}}$. It corresponds to the discontinuity existing in the crisp case when $x$ and $y$ belong to parts that become disconnected. Further work aims at exploiting features of fuzzy set theory in order to avoid this discontinuity, if this is found desirable.

\section{Fuzzy geodesic balls in a fuzzy set}

Since several definitions of fuzzy geodesic distances exist or could be further proposed, we keep the following definitions of fuzzy geodesic balls as general as possible. Therefore, all what follows can be applied for any definition of a fuzzy geodesic distance, as a crisp number or as a fuzzy number.

\subsection{General definition}

In this section, we define fuzzy geodesic balls in a fuzzy set. Let us denote by $\beta_{\mu}(x, \rho)$ the fuzzy geodesic ball of center $x$ and radius $\rho$, conditionally to $\mu$. We define $\beta_{\mu}(x, \rho)$ as a fuzzy set on $\mathscr{S}$, and $\beta_{\mu}(x, \rho)(y)$ denotes the membership value of any point $y$ of $\mathscr{S}$ to the fuzzy geodesic ball. Intuitively, given that $x$ is in $\mu$ to some degree, for each point $y$ the value $\beta_{\mu}(x, \rho)(y)$ represents the fact that $y$ belongs to $\mu$ to some degree and that it is at a geodesic distance in $\mu$ from $x$ less than $\rho$. For that, $\beta_{\mu}(x, \rho)(y)$ is defined as a conjunction of three terms: the degree to which $x$ belongs to $\mu$, the degree to which $y$ belongs to $\mu$, and the degree $\delta\left(d_{\mu}(x, y) \leqslant \rho\right)$ to which $d_{\mu}(x, y) \leqslant \rho$, i.e.:

$\forall y \in \mathscr{S}, \beta_{\mu}(x, \rho)(y)=t\left[\mu(x), \mu(y), \delta\left(d_{\mu}(x, y) \leqslant \rho\right)\right]$,

where $t$ is a t-norm. 


\subsection{Simple example}

Obviously, $\delta\left(d_{\mu}(x, y) \leqslant \rho\right)$ should be a decreasing function of $d_{\mu}(x, y)$. If we consider that $d_{\mu}$ and $\rho$ are crisp numbers, we can choose a simple Heaviside function, such that

$\delta\left(d_{\mu}(x, y) \leqslant \rho\right)= \begin{cases}1 & \text { if } d_{\mu}(x, y) \leqslant \rho, \\ 0 & \text { else. }\end{cases}$

Then we derive

$\forall y \in \mathscr{S}, \beta_{\mu}(x, \rho)(y)= \begin{cases}t[\mu(x), \mu(y)] & \text { if } d_{\mu}(x, y) \leqslant \rho, \\ 0 & \text { else. }\end{cases}$

A fuzzy ball is therefore a subset of $\mu$ constituted of points $y$ which are at a geodesic distance from $x$ less than $\rho$, and whose membership degrees are bounded by $\mu(x)$.

In this case, we assume that the value of interest $\rho$ is precisely defined, which may appear as restrictive in a fuzzy context.

\subsection{Comparison of two fuzzy numbers}

If we consider that some imprecision is attached to $\rho$, rather than considering it as crisp, then we can choose a smoother function, depending on the amount of imprecision attached to $\rho$. The problem with this approach is that the chosen decreasing function is somewhat arbitrary, and probably difficult to tune for specific applications.

Therefore, we propose another approach, where the link between this function and the imprecision of $\rho$ is made more explicit. For this aim, we consider $\rho$ as a fuzzy number. Defining $\delta\left(d_{\mu}(x, y) \leqslant \rho\right)$ calls then for the comparison of fuzzy numbers: $d_{\mu}(x, y)$ is less than $\rho$ if $d_{\mu}(x, y)$ is equal to the minimum of $d_{\mu}(x, y)$ and $\rho$. The minimum between two fuzzy numbers has been defined in Ref. [22] as follows. Let $d$ and $\rho$ be two fuzzy numbers. From the definition of fuzzy numbers, the $\alpha$-cuts of $d$ and $\rho$ are bounded intervals, denoted as $\left[d_{\alpha}^{-}, d_{\alpha}^{+}\right]$and $\left[\rho_{\alpha}^{-}, \rho_{\alpha}^{+}\right]$, respectively. The minimum of $d$ and $\rho$ is then the fuzzy number, the $\alpha$-cuts of which are

$\min (d, \rho)_{\alpha}=\left[\min \left(d_{\alpha}^{-}, \rho_{\alpha}^{-}\right), \min \left(d_{\alpha}^{+}, \rho_{\alpha}^{+}\right)\right]$.

Let us denote by $\left[\rho_{0}, \rho_{2}\right]$ the support of $\rho$ and by $\rho_{1}$ its modal value. We use similar notations for $d$. Four configurations are illustrated in Fig. 3, corresponding to different rankings of $\rho_{1}$ and $d_{1}, \rho_{2}$ and $d_{2}, \rho_{3}$ and $d_{3}$. The four other possible configurations can be easily deduced by symmetry (by exchanging the roles of $d$ and $\rho$ ).


Fig. 3. Minimum of two fuzzy numbers $d$ and $\rho$ (thick dashed line). Top left: $d_{1}<\rho_{1}, d_{2}<\rho_{2}, d_{3}<\rho_{3}$, the minimum is equal to $d$. Top right: $d_{1}<\rho_{1}, d_{2}>\rho_{2}, d_{3}<\rho_{3}$, the minimum is equal to $d$ until the first intersection between $d$ and $\rho$, then it is equal to $\rho$ until the third intersection, and then equal to $d$ again. Bottom left: $d_{1}<\rho_{1}, d_{2}<\rho_{2}, d_{3}>\rho_{3}$, the minimum is equal to $d$ until the second intersection, and then to $\rho$. Bottom right: $d_{1}<\rho_{1}, d_{2}>\rho_{2}, d_{3}>\rho_{3}$, the minimum is equal to $d$ until the first intersection, and then equal to $\rho$. 


\subsection{Detailed expression for the geodesic distance defined as a number}

Let us detail the analytical expression of $\delta\left(d_{\mu}(x, y) \leqslant \rho\right)$ in the case where the fuzzy geodesic distance is defined as a crisp number. Applying Eq. (12) in in the case where $d_{\mu}(x, y)$ is a crisp number, we come up with the following result, for all real number $z$ :

- if $d_{\mu}(x, y) \leqslant \rho_{0}$ :

$\min \left(d_{\mu}(x, y), \rho\right)(z)= \begin{cases}1 & \text { if } z=d_{\mu}(x, y), \\ 0 & \text { if } z \neq d_{\mu}(x, y),\end{cases}$

- if $\rho_{0} \leqslant d_{\mu}(x, y) \leqslant \rho_{1}$ :

$\min \left(d_{\mu}(x, y), \rho\right)(z)= \begin{cases}\rho(z) & \text { if } z<d_{\mu}(x, y), \\ 1 & \text { if } z=d_{\mu}(x, y), \\ 0 & \text { if } z>d_{\mu}(x, y),\end{cases}$

- if $\rho_{1} \leqslant d_{\mu}(x, y) \leqslant \rho_{2}$ :

$\min \left(d_{\mu}(x, y), \rho\right)(z)= \begin{cases}\rho(z) & \text { if } z \leqslant d_{\mu}(x, y), \\ 0 & \text { if } z>d_{\mu}(x, y),\end{cases}$

- if $d_{\mu}(x, y) \geqslant \rho_{2}$ :

$\min \left(d_{\mu}(x, y), \rho\right)(z)=\rho(z)$.

To have $d_{\mu}(x, y) \leqslant \rho$ is equivalent to have $d_{\mu}(x, y)=\min \left(d_{\mu}(x, y), \rho\right), \quad$ or $\quad d_{\mu}(x, y) \subset \min \left(d_{\mu}(x, y), \rho\right)$ and $\min \left(d_{\mu}(x, y), \rho\right) \subset d_{\mu}(x, y)$. This last form can be easily translated into fuzzy terms, in a way similar to the one used in Ref. [2], as

$\delta\left(d_{\mu}(x, y) \leqslant \rho\right)$

$=t\left[\inf _{z} T\left[c\left(d_{\mu}(x, y)\right)(z), \min \left(d_{\mu}(x, y), \rho\right)(z)\right]\right.$,

$\left.\inf _{z} T\left[d_{\mu}(x, y)(z), c\left(\min \left(d_{\mu}(x, y), \rho\right)\right)\right]\right]$,

where $t$ is a t-norm, $c$ a fuzzy complementation (typically $c(z)=1-z)$ and $T$ a t-conorm, dual of $t$ with respect to $c$. This leads to the following result:

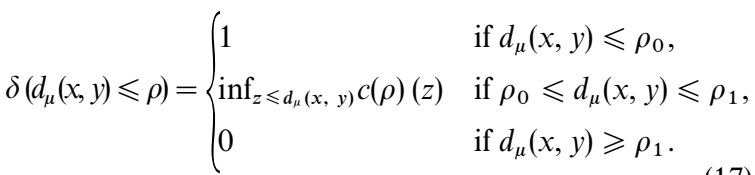

Since $\rho$ is increasing on $\left[\rho_{0}, \rho_{1}\right]$ (as it is a fuzzy number), we obtain

$$
\delta\left(d_{\mu}(x, y) \leqslant \rho\right)= \begin{cases}1 & \text { if } d_{\mu}(x, y) \leqslant \rho_{0}, \\ c(\rho)\left(d_{\mu}(x, y)\right) & \text { if } \rho_{0} \leqslant d_{\mu}(x, y) \leqslant \rho_{1}, \\ 0 & \text { if } d_{\mu}(x, y) \geqslant \rho_{1},\end{cases}
$$

Finally, we obtain

$$
\begin{aligned}
\forall y & \in \mathscr{S}, \beta_{\mu}(x, \rho)(y) \\
& = \begin{cases}0 & \text { if } d_{\mu}(x, y) \geqslant \rho_{1}, \\
t\left[\mu(x), \mu(y), c(\rho)\left(\delta\left(d_{\mu}(x, y)\right)\right]\right. & \text { else. }\end{cases}
\end{aligned}
$$

If $t$ is chosen for instance as the product, $\mu(x)$ and $\mu(y)$ appear as weighting factors.

This definition may appear as severe. For instance, values that are a little bit smaller than $\rho_{1}$ have very low degrees of being less than $\rho$, although they are less than the modal value of $\rho$.

A more "optimistic" definition can be derived from the relationship "to the left of", as introduced in Ref. [26], but applied here in a simpler 1D case. In this approach, we define in the considered space a "fuzzy landscape" representing, for each point, the degree to which this point is in a direction $\mathbf{u}$ from a reference set or a fuzzy set. Here, the space is one-dimensional, and equal to $\mathbb{R}^{+}$. The reference fuzzy set is $\rho$. The direction corresponding to the relationship "to the left of " is the opposite of the unit vector on the real line (horizontal line in Fig. 4). According to the definitions provided in the general case in Ref. [27], the degree to which a point $P$ is to the left of $\rho$ is defined as

$\mu_{\text {left }}(\rho)(P)=\max _{Q} t[\rho(Q), f(\theta(P, Q))]$,

where $t$ is a t-norm, $f$ is a decreasing function in $[0, \pi]$, with $f(0)=1$ and $f(\theta)=0$ for $\theta \geqslant \pi / 2$, and $\theta$ is defined as

$\theta(P, Q)=\arccos \left(\frac{\mathbf{Q P} \cdot \mathbf{u}}{\|\mathbf{Q P}\|}\right)$

and $\theta(P, P)=0$.

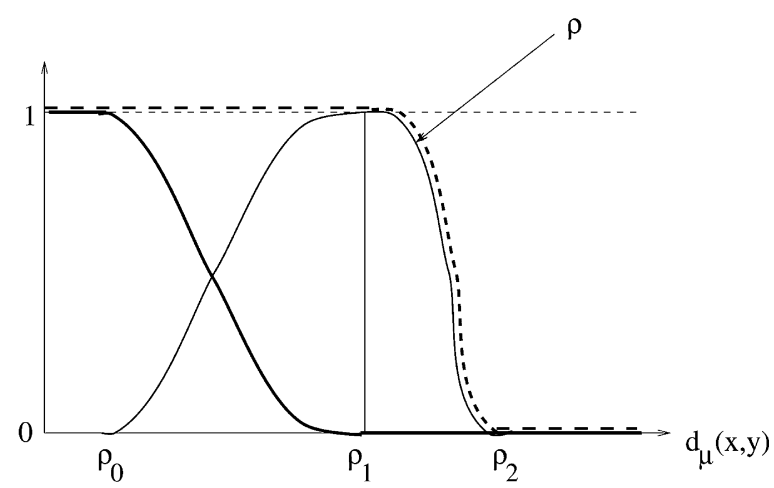

Fig. 4. Illustration of the definition of $\delta\left(d_{\mu}(x, y) \leqslant \rho\right)$ using the minimum of two fuzzy numbers (continuous dark line) and using the relation "left to" (dashed line). 
Let $x_{P}$ and $x_{Q}$ be the coordinates of $P$ and $Q$ on the horizontal axis. We have QP.u $=x_{Q}-x_{P}$, and therefore

$$
\theta(P, Q)= \begin{cases}0 & \text { if } x_{Q}>x_{P} \\ \pi & \text { if } x_{Q}<x_{P}\end{cases}
$$

The first case corresponds to $P$ being on the left of $Q$ and the second one to $P$ being on the right of $Q$. These results lead to the following expression of $\mu_{\text {left }}(\rho)(P)$ :

$$
\mu_{\text {left }}(\rho)(P)=\max _{x_{Q}>x_{P}} \rho\left(x_{Q}\right)
$$

It leads to

$$
\delta\left(d_{\mu}(x, y) \leqslant \rho\right)= \begin{cases}1 & \text { if } d_{\mu}(x, y) \leqslant \rho_{1}, \\ \rho\left(d_{\mu}(x, y)\right) & \text { if } d_{\mu}(x, y) \geqslant \rho_{1} .\end{cases}
$$

These definitions are illustrated in Fig. 4.

The proposed definition of a fuzzy geodesic ball applies directly to any other definition of the fuzzy geodesic distance, represented either as a crisp number, or as a fuzzy number. Also the following properties hold, and are not restricted to the particular form of the fuzzy geodesic distance we use.

\subsection{Properties}

The proposed definitions of fuzzy geodesic balls share the following properties:

(1) $\beta_{\mu}(x, \rho)(x)=\mu(x)$ (since $\delta\left(d_{\mu}(x, x) \leqslant \rho\right)=1$, and 1 is unit element of any t-norm);

(2) $\beta_{\mu}(x, \rho)(y) \leqslant \mu(x)$ (since for any t-norm, we have $\left.\forall(a, b) \in[0,1]^{2}, t(a, b) \leqslant a \wedge t(a, b) \leqslant b\right) ;$

(3) $\beta_{\mu}(x, \rho)(y) \leqslant \mu(y)$;

(4) if $d_{\mu}(x, y)$ and $\rho$ are crisp numbers, $\delta\left(d_{\mu}(x, y) \leqslant \rho\right)$ is binary, and equal to 1 iff $d_{\mu}(x, y) \leqslant \rho$ (by construction);

(5) if $\mu, d_{\mu}$ and $\rho$ are crisp, then $\beta_{\mu}(x, \rho)$ is the crisp geodesic ball, therefore compatibility with the binary case is achieved (this comes from the limit values taken by any t-norm, that correspond exactly to a binary intersection: $t(0,1)=t(1,0)=t(0,0)=0$ and $t(1,1)=1)$

(6) spatial invariance: $\beta_{\mu}(x, \rho)$ is invariant by translation and rotation;

(7) monotony with respect to $\rho$ : if $\rho$ and $\rho^{\prime}$ are such that $\rho_{1} \leqslant \rho^{\prime}{ }_{1}$ and $\rho \leqslant \rho^{\prime}$ on $\left[\rho_{0}, \rho_{1}\right]$ (which is typically the case if $\rho^{\prime}$ is just a translation of $\rho$ ), then $\beta_{\mu}(x, \rho) \leqslant \beta_{\mu}\left(x, \rho^{\prime}\right)$, expressing that a fuzzy geodesic ball is included in a fuzzy geodesic ball of same center and "larger" radius;

(8) a fuzzy geodesic ball is always included in the Euclidean ball of same radius.
These properties are the fuzzy equivalents of the properties of crisp geodesic balls. This shows the consistency of the proposed extension.

\section{Fuzzy geodesic mathematical morphology}

In order to extend geodesic morphological operations to fuzzy sets, we translate Eqs. (2) and (3) into fuzzy terms. The idea is to replace formally every binary concept by its fuzzy equivalent, as presented in Section 2.

\subsection{Definitions of basic fuzzy geodesic operators}

In the geodesic case, we use similar rules as in Section 2 to translate Eqs. (2) and (3) into fuzzy terms. This leads to the following definitions of fuzzy geodesic dilation and erosion of $\mu^{\prime}$ conditionally to $\mu$ :

$$
\begin{aligned}
& \forall x \in \mathscr{S}, D_{\mu}^{\rho}\left(\mu^{\prime}\right)(x)=\sup _{y \in \mathscr{S}} t\left[\beta_{\mu}(x, \rho)(y), \mu^{\prime}(y)\right], \\
& \forall x \in \mathscr{S}, E_{\mu}^{\rho}\left(\mu^{\prime}\right)(x)=\inf _{y \in \mathscr{S}} T\left[c\left(\beta_{\mu}(x, \rho)(y)\right), \mu^{\prime}(y)\right] .
\end{aligned}
$$

From these two basic operators, other ones can be defined, as is done in classical morphology. For instance fuzzy geodesic opening and closing are simply defined as $O_{\mu}^{\rho}\left(\mu^{\prime}\right)=D_{\mu}^{\rho}\left[E_{\mu}^{\rho}\left(\mu^{\prime}\right)\right]$ and $C_{\mu}^{\rho}\left(\mu^{\prime}\right)=E_{\mu}^{\rho}\left[D_{\mu}^{\rho}\left(\mu^{\prime}\right)\right]$.

\subsection{Properties}

The proposed definitions of fuzzy geodesic dilation and erosion have the following properties, which are similar to the properties of classical geodesic operators:

(1) compatibility with the crisp case: if $\mu, \mu^{\prime}$ and $\rho$ are crisp, the definitions are equivalent to the binary geodesic operators;

(2) duality with respect to complementation:

$\forall x \in \mathscr{S}, D_{\mu}^{\rho}\left[c\left(\mu^{\prime}\right)\right](x)=c\left[E_{\mu}^{\rho}\left(\mu^{\prime}\right)\right](x)$

assuming that the t-norm and the t-conorm used in dilation and erosion, respectively, are dual with respect to the complementation $c$;

(3) the result of the geodesic dilation of $\mu^{\prime}$ conditionally to $\mu$ is included in $\mu$ :

$\forall x \in \mathscr{S}, D_{\mu}^{\rho}\left(\mu^{\prime}\right)(x) \leqslant \mu(x)$

expressing that the transformed set stays inside the conditioning set;

(4) invariance with respect to geometrical transformations, and local knowledge property;

(5) increasingness:

$\mu^{\prime} \leqslant \mu^{\prime \prime} \Rightarrow \forall x \in \mathscr{S}, D_{\mu}^{\rho}\left(\mu^{\prime}\right)(x) \leqslant D_{\mu}^{\rho}\left(\mu^{\prime \prime}\right)(x) ;$ 
(6) restricted extensivity:

$$
\forall x \in \mathscr{S}, D_{\mu}^{\rho}\left(\mu^{\prime}\right)(x) \geqslant t\left[\mu(x), \mu^{\prime}(x)\right] ;
$$

(7) interpretation: rewriting the expression of fuzzy geodesic dilation leads to

$$
D_{\mu}^{\rho}\left(\mu^{\prime}\right)(x)=\sup _{y \in \mathscr{S}} t\left[t\left[\mu(x), \mu(y), \delta\left(d_{\mu}(x, y) \leqslant \rho\right)\right], \mu^{\prime}(y)\right]
$$

and, since a t-norm is commutative, associative and increasing

$$
D_{\mu}^{\rho}\left(\mu^{\prime}\right)(x)=t\left[\mu(x), \sup _{y \in \mathscr{S}} t\left[\mu(y), \mu^{\prime}(y), \delta\left(d_{\mu}(x, y) \leqslant \rho\right)\right]\right] .
$$

This represents the intersection of $\mu$ with the dilation of $\mu^{\prime}$ performed on a neighborhood containing the points $y$ of $\mu$ (the conditioning aspect) such that $d_{\mu}(x, y) \leqslant \rho$ (the geodesic distance aspect). This interpretation is in complete agreement with what is expected from a geodesic dilation.

\section{Conclusion}

We presented in this paper an original way to define fuzzy geodesic morphological operators, based on fuzzy geodesic distance. We proposed definitions of these operators and of fuzzy geodesic balls that have good features: they deal with a direct representation of spatial imprecision in the fuzzy sets, they are consistent with existing binary definitions, they have good formal properties, in agreement with the formal properties of crisp definitions and with intuitive requirements.

Future works aim at investigating further properties of these definitions, at comparing the different possible instantiations of them, and at evaluating their applications in image processing problems under imprecision.

\section{References}

[1] I. Bloch, H. Maître, Constructing a fuzzy mathematical morphology: alternative ways, Second IEEE International Conference on Fuzzy Systems, FUZZ IEEE 93, San Fransisco, California, March 1993, pp. 1303-1308.

[2] I. Bloch, H. Maître, Fuzzy mathematical morphologies, a comparative study, Pattern Recognition 28 (9) (1995) 1341-1387.

[3] D. Sinha, E. Dougherty, Fuzzy Mathematical Morphology, J. Visual Commun. Image Representation 3 (3) (1992) 286-302.

[4] B. De Baets, Idempotent closing and opening operations in fuzzy mathematical morphology, ISUMA-NAFIPS'95, College Park, MD, September 1995, pp. 228-233.

[5] V. di Gesu, M.C. Maccarone, M. Tripiciano, Mathematical Morphology based on Fuzzy Operators, in: R. Lowen, M. Roubens (Eds.), Fuzzy Logic, Kluwer Academic, Dordrecht, 1993, pp. 477-486.
[6] A.T. Popov, Morphological operations on fuzzy sets, in: IEE Image Processing and its Applications, Edinburgh, UK, July 1995, pp. 837-840.

[7] D. Sinha, P. Sinha, E.R. Dougherty, S. Batman, Design and analysis of fuzzy morphological algorithms for image processing, IEEE Trans. Fuzzy Systems 5 (4) (1997) 570-584.

[8] I. Bloch, Distances in fuzzy sets for image processing derived from fuzzy mathematical morphology (invited conference), Information Processing and Management of Uncertainty in Knowledge-Based Systems, Granada, Spain, July 1996, pp. 1307-1312.

[9] I. Bloch, On links between fuzzy morphology and fuzzy distances: euclidean and geodesic cases (invited conference), in: Information Processing and Management of Uncertainty IPMU'98, Paris, 1998, pp. 1144-1151.

[10] I. Bloch, Fuzzy morphology and fuzzy distances: new definitions and links in both euclidean and geodesic cases, in: A. Ralescu (Ed.), Lecture Notes in Artificial Intelligence: Fuzzy Logic in Artificial Intelligence, towards Intelligent Systems, Springer, Berlin, 1998.

[11] J. Serra, Image Analysis and Mathematical Morphology, Academic Press, London, 1982.

[12] J. Serra, in: J. Serra (Ed.), Image Analysis and Mathematical Morphology, Part II: Theoretical Advances, Academic Press, London, 1988.

[13] M. Schmitt, J. Mattioli, Morphologie Mathématique, Masson, Paris, 1994

[14] C. Lantuejoul, F. Maisonneuve, Geodesic methods in image analysis. pattern recognition 17 (2) (1984) 177-187.

[15] I. Bloch, Fuzzy geodesic mathematical morphology from fuzzy geodesic distance, in: H. Heijmans, J. Roerdink (Eds.), Mathematical Morphology and its Applications to Image and Signal Processing, Kluwer Academic, Amsterdam, 1998, pp. 43-50.

[16] I. Bloch, Fuzzy Geodesic Distance in Images, in: A. Ralescu, T. Martin (Eds), Lecture Notes in Artificial Intelligence: Fuzzy Logic in Artificial Intelligence, towards Intelligent Systems, Springer, Berlin, 1996, pp. 153-166.

[17] L.A. Zadeh, Fuzzy sets, Inform. and Control 8 (1965) 338-353.

[18] I. Bloch, Image information processing using fuzzy sets (invited conference), World Automation Congress, Soft Computing with Industrial Applications, Montpellier, France, May 1996, pp. 79-84.

[19] L.A. Zadeh, The concept of a linguistic variable and its application to approximate reasoning, Inform. Sci. 8 (1975) 199-249.

[20] I. Bloch, H. Maître, M. Anvari, Fuzzy adjacency between image objects, Int. J. Uncertainty, Fuzziness KnowledgeBased Systems 5 (6) (1997) 615-653.

[21] I. Bloch, On fuzzy distances and their use in image processing under imprecision. Pattern Recognition 32 (11) (1999) 1873-1895.

[22] D. Dubois, H. Prade, Fuzzy Sets and Systems: Theory and Applications, Academic Press, New-York, 1980.

[23] D. Dubois, H. Prade, A review of fuzzy set aggregation connectives, Inform. Sci. 36 (1985) 85-121.

[24] R.R. Yager, On a general class of fuzzy connectives, Fuzzy Sets and Systems 4 (1980) 235-242.

[25] A. Rosenfeld, The fuzzy geometry of image subsets, Pattern Recognition Lett. 2 (1984) 311-317. 
[26] I. Bloch, Fuzzy relative position between objects in images: a morphological approach, IEEE Interational Conference on Image Processing ICIP'96, Vol. II, Lausanne, September 1996, pp. 987-990.
[27] I. Bloch, Fuzzy relative position between objects in image processing: a morphological approach, IEEE Trans. Pattern Anal. Mach. Intell. 21(5) (1999).

\begin{abstract}
About the Author-ISABELLE BLOCH is professor at ENST Paris (Signal and Image Department), and is in charge of the Image Processing and Interpretation Group. She graduated from Ecole des Mines de Paris in 1986, received a Ph.D. from ENST Paris in 1990, and the "Habilitation à Diriger des Recherches" from University Paris 5 in 1995. Her research interests include 3D image and object processing, structural pattern recognition, 3D and fuzzy mathematical morphology, decision theory, data fusion in image processing, fuzzy set theory, evidence theory, medical imaging, aerial and satellite imaging.
\end{abstract}

